\title{
Large-scale photospheric motions determined from granule tracking and helioseismology from SDO/HMI data
}

\author{
Th. Roudier ${ }^{1}$, M. Švanda ${ }^{2,3}$, J. Ballot ${ }^{1}$, J. M. Malherbe ${ }^{4}$, and M. Rieutord ${ }^{1}$ \\ ${ }^{1}$ Institut de Recherche en Astrophysique et Planétologie, Université de Toulouse, CNRS, UPS, CNES, 14 avenue Edouard Belin, \\ 31400 Toulouse, France \\ e-mail: thierry.roudier@irap.omp.eu \\ 2 Astronomical Institute (v. v. i.), Czech Academy of Sciences, Fričova 298, 25165 Ondřejov, Czech Republic \\ ${ }^{3}$ Charles University, Astronomical Institute, Faculty of Mathematics and Physics, V Holešovičkách 2, 18000 Prague 8, \\ Czech Republic \\ ${ }^{4}$ LESIA, Observatoire de Paris, Section de Meudon, 92195 Meudon, France
}

Received 28 September 2017 / Accepted 5 December 2017

\begin{abstract}
Context. Large-scale flows in the Sun play an important role in the dynamo process linked to the solar cycle. The important large-scale flows are the differential rotation and the meridional circulation with an amplitude of $\mathrm{km} \mathrm{s}^{-1}$ and few m s${ }^{-1}$, respectively. These flows also have a cycle-related components, namely the torsional oscillations.

Aims. Our attempt is to determine large-scale plasma flows on the solar surface by deriving horizontal flow velocities using the techniques of solar granule tracking, dopplergrams, and time-distance helioseismology.

Methods. Coherent structure tracking (CST) and time-distance helioseismology were used to investigate the solar differential rotation and meridional circulation at the solar surface on a 30-day HMI/SDO sequence. The influence of a large sunspot on these large-scale flows with a specific 7-day HMI/SDO sequence has been also studied.

Results. The large-scale flows measured by the CST on the solar surface and the same flow determined from the same data with the helioseismology in the first $1 \mathrm{Mm}$ below the surface are in good agreement in amplitude and direction. The torsional waves are also located at the same latitudes with amplitude of the same order. We are able to measure the meridional circulation correctly using the CST method with only 3 days of data and after averaging between $\pm 15^{\circ}$ in longitude.

Conclusions. We conclude that the combination of CST and Doppler velocities allows us to detect properly the differential solar rotation and also smaller amplitude flows such as the meridional circulation and torsional waves. The results of our methods are in good agreement with helioseismic measurements.
\end{abstract}

Key words. Sun: atmosphere - Sun: granulation - Sun: helioseismology - Sun: rotation

\section{Introduction}

Large-scale motions in the solar convection zone are important elements to understand the evolution of solar magnetism. Various methods (Paternò 2010), based on helioseismology (Gizon \& Rempel 2008; Komm et al. 2014; Zhao et al. 2014), Doppler velocities measurements (Duvall 1979; Hathaway 1987), solar magnetic tracers (e.g. sunspots, sunspot groups, faculae, bright points; see Hathaway \& Upton 2014) or surface feature (e.g. supergranule) tracking (Švanda et al. 2008; Hathaway 2012; Löptien et al. 2017) are used to infer the large-scale solar flows. Advantages and disadvantages of various techniques are discussed and summarized by Hathaway \& Upton (2014).

In particular, magnetic features do not behave as ideal tracers because of their interactions with the surrounding plasma (Paternò 2010). The use of proxies may induce measurement biases. For example, the velocities measured using sunspots or faculae as tracers presumably reflect the properties of deeper layers and therefore do not give access to the actual surface plasma motions (Javaraiah 2013; Li et al. 2013). It is well establish now that rotation deduced from motion of sunspots is systematically faster than that deduced from spectroscopic observations (Hathaway \& Upton 2014). This is partly because the concentrated magnetic field, which serves as a proxy, is anchored in the underlying solar plasma; both the magnetic field and the solar plasma interact strongly in the convective zone, where the plasma $\beta$ parameter, which is the ratio of the plasma pressure to the magnetic pressure $\left(\beta=2 \mu P / B^{2}\right)$, is large. In this way, the study of the movements of proxies is delicate (Sudar et al. 2017) because observational biases can lead to contradictory results, such as in the measurement of the meridional circulation, the amplitude of which is only about $10 \mathrm{~m} \mathrm{~s}^{-1}$. Helioseismology currently provides the best measurement inside the Sun up to near the surface of the plasma flows [from 30 to $1 \mathrm{Mm}$ ] (Howe 2009; Zhao 2016).

One of the attempts to determine plasma motions on the surface of the full-disc Sun was made by Švanda et al. (2008) and in a recent work by Löptien et al. (2017) using local correlation tracking (LCT; November 1986). These works detect reliable motion of features that are carried by an underlying larger scale velocity field. They also show that a detection of torsional waves and meridional circulation is possible by applying the LCT method to MDI/SOHO and HMI/SDO Dopplergrams and continuum intensity data, respectively. Nevertheless, the LCT method is subject to limitations when applied to whole Sun data. This technique is sensitive to the distance to the centre initially 
because the properties (e.g. size and contrast) of the granules change because of projection effects; secondly, the so-called shrinking effect due to the apparent asymmetry of granulation depends on the viewing angle inclined from the vertical, in combination with the expansion of the granules, gives an apparent motion towards the disc centre (Löptien et al. 2016).

Moreover, the LCT is well known for underestimating the amplitude of the velocities by a factor that depends on a particular application and may reach up to factor 2 or 3 (Verma et al. 2013).

We propose applying the coherent structure tracking (CST; Rieutord et al. 2007; Roudier et al. 2012) code to follow the proper motions of solar granules in full-disc HMI/SDO data. By measuring the flow field on the solar surface over a long time period we obtain a representative description of solar plasma evolution. The CST allows us to get flow field from covering the spatial scales from $2.5 \mathrm{Mm}$ up to nearly $85 \%$ of the solar radius. This method is complementary to helioseismic methods of flow determination, which describes the flows below the solar surface. The CST is a granule tracking technique, which allows us to estimate the field direction and amplitude (Rieutord et al. 2007). The LCT and Fourier local correlation tracking (FLCT; Welsch et al. 2004; Fisher \& Welsch 2008) account for both granules and intergranules when cross-correlating continuum images to estimate the direction and amplitude of the field. The principal difference between the LCT (FLCT) and CST techniques is that the LCT (FLCT) evaluates the similarity of image subframes at different positions in different times. These subframes are required to contain distinct features (granules, supergranule, etc.), but these features are not uniquely identified. Thus the subframe displacement is evaluated based on an overall similarity. The LCT (FLCT) thus provides a smooth differentiable estimate of the velocity field. In CST, the code identifies individual features (granules) and tracks these individual features coherently through out the image sequence. The resulting velocity field is thus discontinuous and the differentiable extension is estimated based on multi-resolution analysis.

In this paper, we describe a comparison between velocity fields on the full Sun obtained by various methods (CST, LCT, FLCT, and time-distance helioseismology). A 30-day sequence of quiet Sun spanning from 16 August to 14 September 2010 is selected. This epoch was particularly selected because the variations of the heliographic latitude of the solar disc centre $\left(B_{0}\right)$ are minimal. This selection allows to us, first, to describe the longterm behaviour of the quiet-Sun surface flows and, second, to avoid possible biases introduced by $B_{0}$ changes, which are mentioned several times in the literature (Liang et al. 2017; Basu \& Antia 2010). We complement this study with the analysis of flows around a large solar spot observed between 12 and 18 April 2016 by $\mathrm{HMI} / \mathrm{SDO}$.

We also discuss the data reduction carried out to get an accurate velocity measurements of the large-scale flows with a low amplitude. We transform the combination the horizontal components measured via the CST and Doppler observations to spherical coordinates suitable for a proper description of the solar differential rotation and residual zonal flow, and meridional circulation. We also study the evolution of the divergence field. Finally, we pay special attention to the effects of a very large sunspot on solar rotation and meridional circulation. The aim of this work is twofold. First, we use solar granules as passive scalars to follow solar plasma motions and, second, we obtain quantitative measurements of large-scale movements at the surface of the Sun.

\section{SDO/HMI observations}

\subsection{SDO/HMI observations}

The Helioseismic and Magnetic Imager (HMI; Scherrer et al. 2012; Schou et al. 2012) on board the Solar Dynamics Observatory (SDO) provides uninterrupted observations of the full disc of the Sun. This provides a unique opportunity to map surface flows on various spatial and temporal scales. We selected the SDO/HMI continuum intensity data from 16 August to 14 September 2010. This period was chosen due to its low solar activity and also to get very small variations of the $B_{0}$ angle. The solar differential rotation and meridional circulation are determined from SDO/HMI continuum intensity and Doppler data taken during this 30-day sequence. A second HMI/SDO time sequence from 12 to 18 April 2016 was used to describe the effects of the flows around a large sunspot to the solar rotation and meridional circulation. Both sequences use the original cadence of $45 \mathrm{~s}$ and the original pixel size of $0 .{ }^{\prime} 5$. The resolution of the HMI instrument is $11^{\prime \prime} 0$.

\subsection{Data analysis}

The flows were measured with two independent methods.

\subsubsection{Granulation tracking by CST}

The CST used the solar granules as passive scalars to follow solar plasma motions. In order to be suitable for the CST application, the data series of the HMI intensitygrams must first be prepared. All frames of the sequence were aligned such that the centre of the solar disc lies exactly on the same pixel in CCD coordinates and the radius of the solar disc was exactly the same for all the frames. The reference values for the position of the disc centre and the radius were obtained from the first image (obtained on 16 August 2010 at 00:00:45 UT) of the 30-day series. Then we performed the granulation tracking using the CST code (Roudier et al. 2012) to reconstruct the projection of the photospheric velocity field $\left(v_{x}, v_{y}\right)$ in the plane of the sky (CCD plane) (Rincon et al. 2017). The application of CST to such a series leads to a sequence of horizontal velocity field maps in the projection to a sky plane with a temporal resolution of $30 \mathrm{~min}$ and a spatial resolution of $2.5 \mathrm{Mm}\left(3^{\prime \prime} .5\right)$, that is the full-disc velocity map has a size of $586 \times 586$ pixel $^{2}$. We further removed the $(x, y)$ velocity signal associated with the motions of the SDO satellite and Earth's orbital displacements from the CST velocity maps following the procedure described by Rincon et al. (2017).

The Dopplergrams provide a key piece of information to reconstruct the full vector field. The HMI convention is that the line-of-sight (l.o.s.) velocity signal $v_{z}$ is taken as positive when the flow is away from the observer, so that the out of plane toward the observer is $v_{\text {dop }}=-v_{z}$ (see Fig. 10 in Roudier et al. 2013). The processing steps of the Dopplergrams were to remove Doppler shift associated with the proper motion of satellite and Earth's displacement from the raw Doppler signal (see Rincon et al. 2017). Then in the Doppler data we corrected a polynomial radial limb shift function adjusted from ring averages of $2 \mathrm{~h}$ of data. Since the $586^{2} \mathrm{px}^{2}$ velocity maps obtained from the CST are limited to an effective resolution of $2.5 \mathrm{Mm}$, we then downsampled the $4096^{2} \mathrm{px}^{2}$ Dopplergrams to the size of CST maps. The downsampled Doppler images were finally averaged over $30 \mathrm{~min}$ to match the temporal sampling of the CST-derived flow maps. 
The velocity induced by the evolution of $B_{0}$ in time $\left(\frac{\mathrm{d} B_{0}}{\mathrm{~d} t}\right)$ was corrected at the step of the correction of the motions of the satellite.

\subsubsection{Time-distance helioseismology}

The surface flows inferred by CST code were compared to flows in the near-surface layers determined by the time-distance helioseismology method (Duvall et al. 1993). Time-distance helioseismology is a set of tools that measure and interpret travel times of seismic waves travelling through the solar interior. Perturbations in the interior cause measurable shifts in travel times, which can be inverted to learn about the origin of these perturbations. Plasma flows are very strong perturbers with a clear imprint in the difference travel times, that is in the difference of travel times of waves propagating between two points in the opposite direction.

We used a data analysis pipeline described in detail in Švanda et al. (2011), which was validated against the direct surface measurements by Švanda et al. (2013). The time-distance pipeline running at Astronomical Institute of the Czech Academy of Sciences consists of codes for data handling, filtering (we routinely use both ridge and phase-speed filters), travel-time measurements, and an inversion process. The travel times are measured and inverted for flows using plane-parallel approximation in a small square patch (about $60^{\circ}$ on a side) near the central meridian in a Postel projection. For this study we focussed on the travel times of the surface gravity $(f$ - $)$ mode with 24-h averaging in time, which sets the random-error levels to $18 \mathrm{~m} \mathrm{~s}^{-1}$ for horizontal components, and did not investigate the vertical flow component.

We performed the flow inversions in three patches around the central meridian for a given date centred at latitudes of $-35^{\circ}, 0^{\circ}$, and $+35^{\circ}$. The patches were then sewn together, in the course of this procedure the horizontal flows were reprojected to a Carrington coordinate system.

The flow maps inferred by our time-distance pipeline have an effective spatial resolution of $10 \mathrm{Mm}$, which is given by the extent of the inversion averaging kernel.

The horizontal flow maps obtained by the CST code were then compared to time-distance $f$-mode near-surface maps on a pixel-to-pixel basis repeating the procedure described in detail in Švanda et al. (2013). The results of this exercise were almost identical to our earlier comparison, that is the correlation coefficient between the CST- and time-distance-derived flows was between 0.7 and 0.8 for all 30 days in the series and both horizontal components. The magnitudes of the flow vectors were also comparable when taking the various effective resolutions into account; time-distance helioseismology underestimated the flow by some $3-5 \%$. However we noticed a systematic offset of the zonal $(x)$ component, when the time-distance flow estimates were systematically by $30-50 \mathrm{~m} \mathrm{~s}^{-1}$ larger. No such offset was noticed in the meridional $(y)$ component of the flow. We assume that the offset in the zonal component is caused by differences in the data processing pipelines, namely in the tracking procedures.

\section{Determination of the solar differential rotation}

\subsection{Solar differential rotation from Dopplergrams}

It is well known that the profile of the differential rotation of the Sun differs by method, data set, and time. However, there has been rotational profiles published in the literature that are considered a reference. A class of the reference profiles was obtained by a spectroscopic method (Paternò 2010), where historically the profile of, for example Howard \& Harvey (1970), is often used.

Following the spectroscopic technique, we obtained our reference solar differential rotation profile from the corrected sequence of Dopplergrams described above.

That rotational profile is computed through the relation

$$
\Omega(\theta)=\frac{v_{\mathrm{dop}}(\theta, \varphi)}{R \cos B_{0} \cos \theta \sin \varphi},
$$

where $\theta$ and $\varphi$ are the latitude and longitude, respectively, and $R$ the solar radius expressed in $\mathrm{km}$. The profile inferred from an average over the 30 days of observation is shown in Fig. 1 (dash-dotted line) and the fit of the polynomial in $\sin \theta$ is given, in $\mu \mathrm{rad} \mathrm{s}^{-1}$, by

$$
\begin{aligned}
\Omega(\theta)= & 2.87+0.0051 \sin \theta-0.529 \sin ^{2} \theta \\
& +0.00124 \sin ^{3} \theta-0.395 \sin ^{4} \theta .
\end{aligned}
$$

The fit was performed in longitudes $\pm 80^{\circ}$ and the corresponding equatorial rotation velocity is $1.998 \pm 0.002 \mathrm{~km} \mathrm{~s}^{-1}$.

This rotational profiles was used as a reference for comparison of the other techniques.

\subsection{Solar differential rotation from CST and Doppler velocity vector}

One of the first scientific applications of the CST algorithm on SDO/HMI data described in Roudier et al. (2013) was to determine the solar rotation from the granule displacements. The horizontal flow $\left(v_{x}, v_{y}\right)$ measured in the plane-of-the-sky coordinates by CST code together with $v_{\text {dop }}$ obtained from corresponding Dopplergrams may be transformed to spherical velocity components $\left(v_{r}, v_{\varphi}, v_{\theta}\right)$. A detailed description of the transform is provided in Roudier et al. (2013), namely in Chapter 5 and Fig. 10.

In our current study, we further projected $v_{\varphi}$ and $v_{\theta}$ to a rectangular $(\varphi, \theta)$ map and, finally, a Carrington map is computed from the entire 30-day sequence. Then the mean profile of rotation is obtained by averaging the flow map in the Carrington coordinates over all longitudes.

The fit of the polynomial in $\sin \theta$ is given, in $\mu \mathrm{rad} \mathrm{s}^{-1}$, by

$$
\begin{aligned}
\Omega_{\varphi}(\theta)= & 2.62+0.0465 \sin \theta-1.70 \sin ^{2} \theta \\
& -0.0177 \sin ^{3} \theta-0.630 \sin ^{4} \theta .
\end{aligned}
$$

It seems that the equatorial rotation obtained from $\Omega_{\varphi}(\theta)$ is $9 \%$ smaller than the reference spectroscopic profile computed from the Dopplergrams. Since spectroscopic profile is our reference, we take the factor of 1.09 to be a correction factor that matches the amplitudes of the two techniques that we use further. Figure 1 shows (solid line) the corrected differential rotation profile. We note that this profile is to be compared to a reference spectroscopic profile described in the preceding subsection and plotted by a dash-dotted line in the same figure. In addition to that amplification factor, the two rotational curves show a very good correspondence in the northern region, but a slight underestimation (around $4 \mathrm{~m} \mathrm{~s}^{-1}$ ) of the solar rotation in the southern region.

The observed asymmetry about the equator seen with the CST is in great part due to the high $B_{0}$ angle around $+7^{\circ}$, which implies a larger projection effect and lower amplitude velocity 
in the south. Such asymmetry was not detected up to $80^{\circ}$ in our first SDO data analysis (see Fig. 6 of Roudier et al. 2013), on 10 December 2011 , where $B_{0}=-0.28^{\circ}$ was very small, reducing the projection effects. The correction for $B_{0}$ evolution described in Sect. 2.2.1 does not take into account the foreshortening of the granules, which introduces a systematic bias in the velocity field. Both components of horizontal velocity are affected by this issue and are the main cause of the observed asymmetry. However, in the equatorial patch this effect is small and an apparently observed asymmetry about the equator is consistent within various methods (e.g. not only CST, but also time-distance).

\subsection{Solar differential rotation from helioseismology}

Similar to the CST method, the differential rotation profile was derived also for the time-distance $f$-mode flow. Since during the stitching of the tiles investigated separately at latitudes $0^{\circ}$ and $\pm 35^{\circ}$ the flow was already transformed to the Carrington coordinates, we only needed to averaged a synoptic map over longitudes and fit the polynomial in $\sin \theta$.

The fit, again in $\mu \mathrm{rad} \mathrm{s}^{-1}$, is

$$
\begin{aligned}
\Omega(\theta)= & 2.84+0.00772 \sin \theta-0.192 \sin ^{2} \theta \\
& -0.0415 \sin ^{3} \theta-0.805 \sin ^{4} \theta .
\end{aligned}
$$

Such a profile gives an equatorial velocity of $1.977 \mathrm{~km} \mathrm{~s}^{-1}$, which is in good agreement with a reference equatorial rotation speed inferred from the spectroscopic method.

The helioseismic profile averaged over the $30 \mathrm{~d}$ of observation is again plotted in Fig. 1. Compared to other techniques, the agreement is very good in equatorial regions and deviates significantly towards larger latitudes; the helioseismic profile is more solid than the other techniques. At $50^{\circ}$ latitude the difference in speeds is almost $200 \mathrm{~m} \mathrm{~s}^{-1}$. This is probably due to the nonlinear effects, pointed out already by Jackiewicz et al. (2007). Flows with magnitudes larger than some $150 \mathrm{~m} \mathrm{~s}^{-1}$ are underestimated by linear helioseismic approach, as beyond this limit even the velocity becomes a strong perturber and the linearity assumption is violated. The zonal flows at large latitudes reach the amplitudes of several hundred $\mathrm{m} \mathrm{s}^{-1}$ compared to the tracking rate, so naturally the non-linearity steps in.

\subsection{Solar differential rotation from divergence maps}

The horizontal velocities $v_{x}$ and $v_{y}$ from CST code allow us to access directly the divergence field over the full Sun with a time step of $30 \mathrm{~min}$ (Fig. 4) in the 30-day sequence. This field reveals the supergranular pattern, which contributes to a diffusion of the magnetic field over the solar surface. To study the field, we first remap the divergence maps using a Sanson-Flamsteed (sinusoidal) projection following the transform

$X=\varphi \cos \theta \quad$ and $\quad Y=\theta$.

The Sanson-Flamsteed projection conserves the areas, which is a necessary requirement for the following steps (Švanda et al. 2006).

From the sequence of divergence maps we infer the apparent velocities by two methods: LCT (November 1986) and FLCT (Welsch et al. 2004; Fisher \& Welsch 2008). Both methods in essence track the features (divergence centres that mark the location of supergranular cells) in a sequence of frames and measure the continuous displacement field that is in the end converted to horizontal velocities in the $(X, Y)$ coordinates.

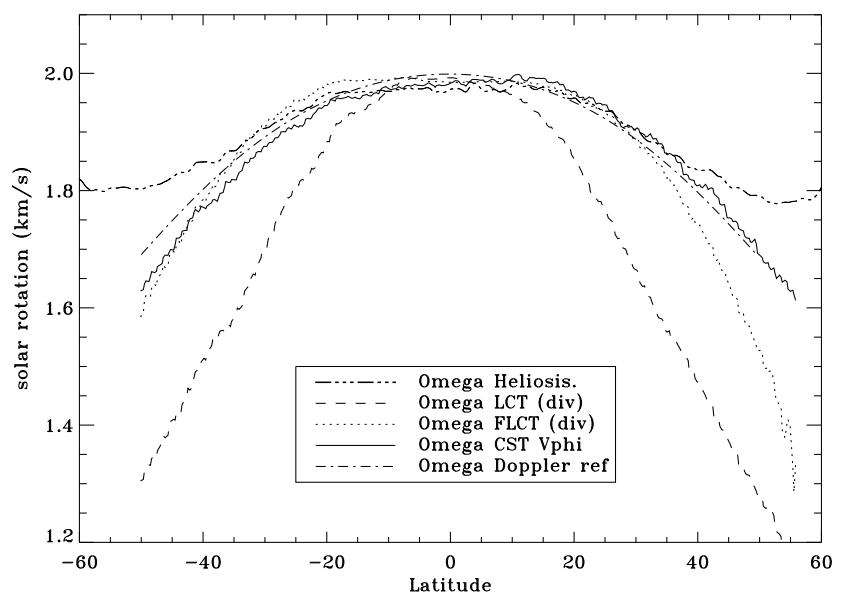

Fig. 1. Solar zonal rotation $\Omega(\theta)$ expressed in $\mathrm{km} \mathrm{s}^{-1}$.

The inferred velocities $v_{X}$ and $v_{Y}$ are then converted to cylindrical coordinates $(\varphi, \theta)$ on a sphere using a transformation

$v_{\varphi}=R\left(v_{X}+\varphi v_{Y} \sin \theta\right)$,

$v_{\theta}=R v_{Y}$

with

$v_{X}=\mathrm{d} X / \mathrm{d} t$,

$v_{Y}=\mathrm{d} Y / \mathrm{d} t$.

As it was carried out for the other methods, $\left(v_{\varphi}, v_{\theta}\right)$ velocities from both LCT and FLCT are projected to Carrington maps for all the 30 days of the sequence. From the synoptic maps we again obtained the averaged flow profiles as a function of the latitude by averaging over longitudes. The differential rotation from LCT and FLCT methods using divergence field as tracers are shown in Fig. 1 (dashed line for LCT and dotted for FLCT). Both tracking methods are known to underestimate the velocities, thus we multiplied these methods by an empirically obtained factors of 1.15 for LCT and 1.065 for FLCT, respectively.

Obviously, the differential rotation curve from LCT differs significantly from the other methods outside the equatorial zone, where a rotation curve is more differential. The FLCT curve agrees very well with a Doppler reference up to $\pm 35^{\circ}$, farther from the equator the velocities are underestimated, particularly in the northern hemisphere. The LCT and FLCT do not measure the same flows as the Doppler measurement does. The LCT and FLCT are sensitive to the divergence and inter-divergence horizontal displacement evolution, whereas the Doppler measurement is affected by its radial location relative to the disc centre. Indeed the Doppler velocity at the disc centre represents vertical flow at $100 \mathrm{~km}$ of altitude and away from that location Doppler velocities are sensitive to the horizontal flows at around $200 \mathrm{~km}$ of altitude (Fleck et al. 2011). This effect is partly corrected by the limb-shift correction. All the methods (LCT, FLCT, Doppler, and CST) are affected by projection effects that mix the granular flows outside the disc centre.

\section{Residual zonal flow}

The solar rotation has a time-varying component, the torsional oscillations (Howard \& Labonte 1980; Kosovichev \& Zhao 2016; Zhao 2016), which can be measured via the residual zonal flow. This component has a very low amplitude (a few $\mathrm{m} \mathrm{s}^{-1}$ ) compared to the magnitude of the solar rotation (almost $2 \mathrm{~km} \mathrm{~s}^{-1}$ at 


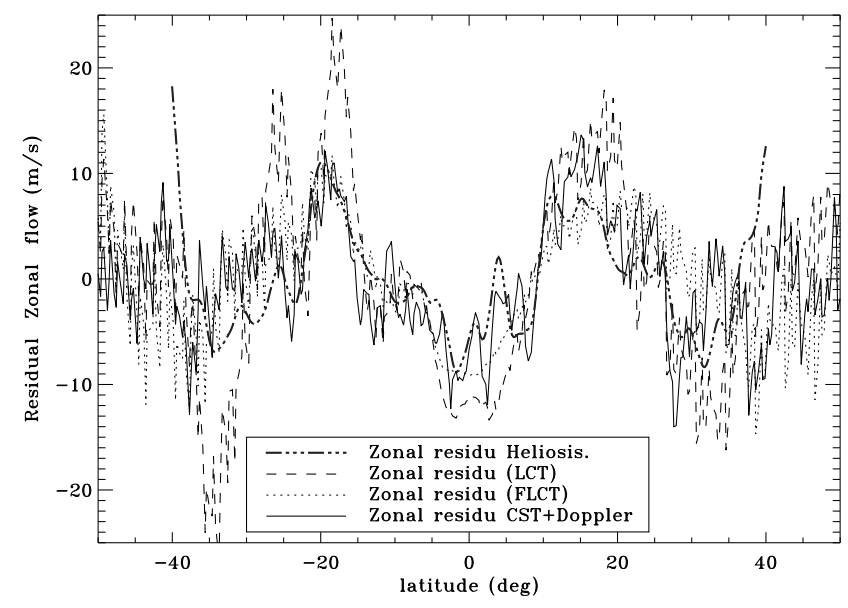

Fig. 2. Solar residual rotation expressed in $\mathrm{m} \mathrm{s}^{-1}$.

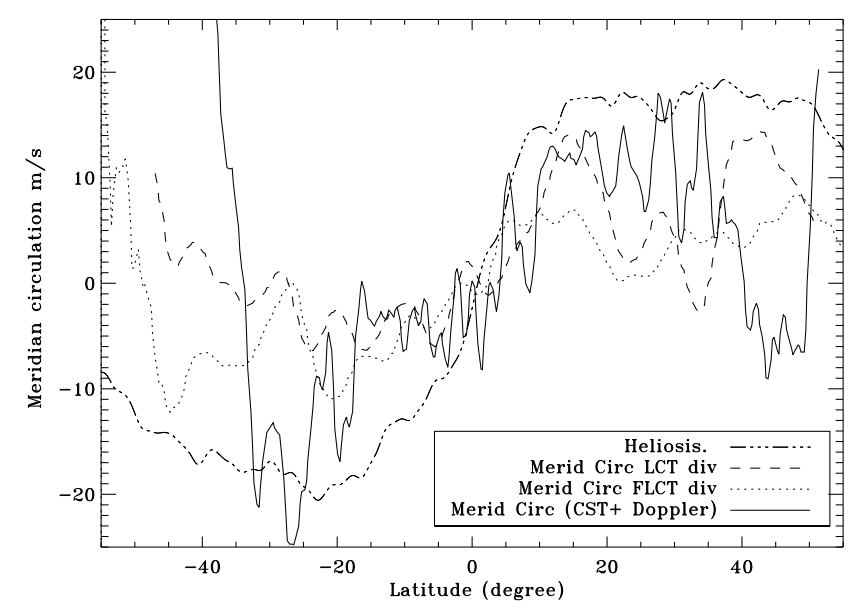

Fig. 3. Meridian circulation expressed in $\mathrm{m} \mathrm{s}^{-1}$.

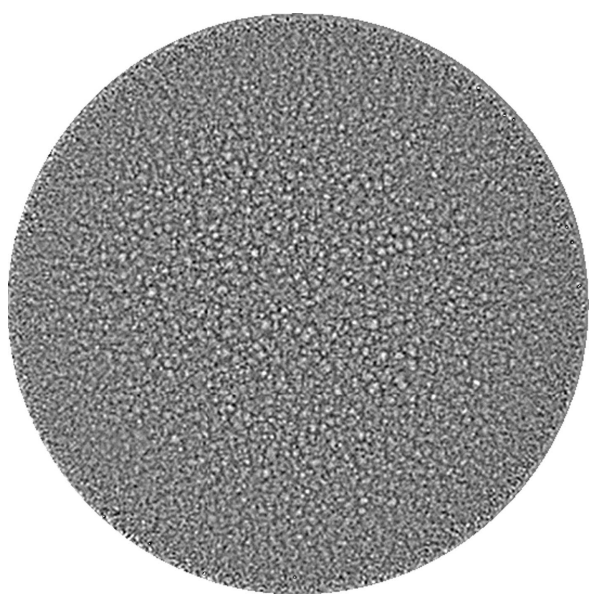

Fig. 4. Divergence map computed from the CST velocities $v_{x}$ and $v_{y}$.

the equator) and needs to be revealed by applying a sufficiently large time averaging window.

In order to obtain the residual flow for a 30-day average, we first fit the averaged zonal flow profile (the differential rotation) by a fourth-degree polynomial in $\sin \theta$ and then subtract this fit from the averaged flow profile. Figure 2 shows the residual zonal flow inferred by various methods of measurement. All the plots show the same behaviour, in which maxima are found around $15^{\circ}$ to $20^{\circ}$ in the northern region (positive latitudes) and around $-20^{\circ}$ in the south region (negative latitudes). The central depression is found at the equator. The amplitude at the maxima are between $\pm 10 \mathrm{~m} \mathrm{~s}^{-1}$. These values, the latitude of the maxima, and their amplitude are in good agreement, at the same date as that found in the literature (e.g. Zhao et al. 2014; Komm et al. 2014; Gizon \& Rempel 2008). The residual zonal flow obtained by the LCT exhibits a larger amplitude of the fluctuation in the southern region relative to the other curves.

The exact appearance of the torsional oscillation pattern is quite sensitive to the background term that is subtracted (see Figs. 24 and 25 in Howe 2009). Two methods are used to reach the detection the torsional oscillation. The first method consists of the subtraction of the rotation profile measured in the quiet (minimum) periods from the profiles in the active periods. Both profiles are averaged over longer times (typically many Carrington rotations) (Howe et al. 2005, 2006). The second method, which is largely consistent with that above, is the subtraction of a smooth fit (usually a polynomial in the sine latitude) from the general profile. The later method is common for time-distance helioseismic results and enables us to study time evolution of the profile (see e.g. Zhao \& Kosovichev 2004). We conclude that the combination of CST and Doppler velocities allows us to detect torsional waves properly. We also note that the results of our methods is in good agreement with helioseismic measurements.

\section{Meridional circulation flow}

The meridional circulation is a long-lived photospheric flow amplitude that is around 10 to $20 \mathrm{~m} \mathrm{~s}^{-1}$ and requires the averaging of measured flows in time. The profile of the meridional flow as a function of latitude is obtained by averaging the meridional $v_{\theta}$ flow component over longitudes, which uses an analogous method as for the determination of the differential rotation, only applied to the other component of the horizontal flow.

Figure 3 shows the profiles of surface meridional circulation obtained from the various methods discussed above. For all the curves we note a detection of the meridional circulation between $-25^{\circ}$ and $+15^{\circ}$ but with a smaller amplitude for the LCT and FLCT methods. The trend seen in the combination of CST horizontal velocities and the Doppler component agrees very well with the trend of the helioseismic determination, the amplitudes of both are more or less similar around $\pm 15 \mathrm{~m} \mathrm{~s}^{-1}$, however the slope seems about twice as steep in case of the helioseismic inference. The velocities determined with CST and Doppler data however do not allow reliable measurements beyond $-40^{\circ}$. This is because of the $B_{0}$ angle (between $6.70^{\circ}$ to $7.22^{\circ}$ ), which induces a large projection effect (foreshortening) of the solar granulation at high latitudes in the southern region. Owing to the foreshortening effect, which is symmetrical about the disc centre, averaging over longitude from the central meridian is limited around $\pm 45^{\circ}$. It is difficult to directly compare anything other than the trends in the case of data set we have at our disposal. For instance, the intrinsic variability of the two profiles has different typical spatial scales, where the profile obtained from the combination of CST and Doppler velocities varies much faster with latitude than the helioseismic profile. A proper study would require a much larger sample of velocities and is beyond the scope of a current study.

By integrating 30 days of data, we are able to recover the surface meridional flow around the equator with the various techniques we mutually compare. The meridional flows are consistent between the methods and clearly show motions towards the poles in both hemispheres. 


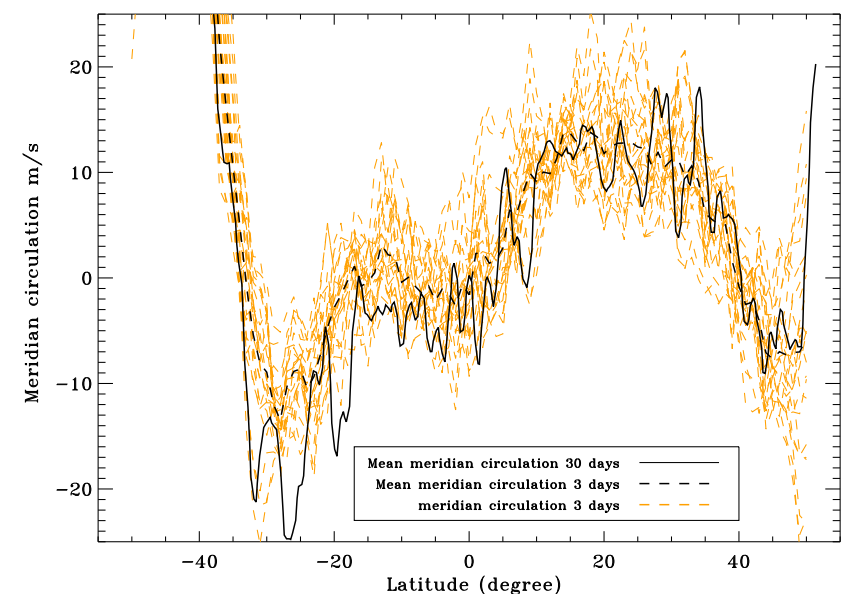

Fig. 5. Meridional circulation measured with a moving temporal window of 3 days during the 30-day sequence (orange dashed line), meridional circulation issued from the temporal averaging of 30 days (solid line) and the average of all the 3-day averages (dashed lines).

Owing to its very small amplitude, the reliable measurement of the meridional circulation flow requires an averaging of a long time sequence (Hathaway 1996; Gizon \& Rempel 2008; Ulrich 2010; Komm et al. 2014; Zhao et al. 2013; Kosovichev \& Zhao 2016; Zhao 2016). We further focus on measurements obtained by a combination of CST horizontal velocities and Doppler velocities. Our goal is to determine how short the temporal window can be to obtain a reasonable signal-to-noise ratio to be able study its evolution during the solar cycle. In the parameter space of a temporal window averaging and longitude averaging domain centred on the central meridian, we tested various configurations of that parameter. The best compromise to minimise the temporal averaging is found for a temporal window of 3 days and a longitudinal averaging between $\pm 15^{\circ}$.

We evaluate the optimal parameter selection by comparing the short-term and longitude span with a 30-day all-longitudes average. For the parameter selection described above the shortterm and longitude span curves differ from the long-term alllongitudes average with a standard deviation of $5 \mathrm{~m} \mathrm{~s}^{-1}$, which is a reasonable value given the fact that the amplitude of the meridional flow is about $15 \mathrm{~m} \mathrm{~s}^{-1}$. In Fig. 5 we plot the longterm all-longitudes average (solid line) together with all 3-day averages along a patch in longitudes of $\pm 15^{\circ}$ around the central meridian (orange dashed lines), and the average of all the 3-day averages (dashed lines). We note that when the plotted 3-day averages are averaged together, they do not agree exactly with the 30-day reference, however the differences are small (around $2-4 \mathrm{~m} \mathrm{~s}^{-1}$ in the south part). That is because the 3-day averages are computed directly from $v_{\varphi}$ maps, whereas the 30-day average is computed from the Carrington synoptic maps.

\section{Effects of a stand-alone sunspot on large-scale flows}

The presence of small sunspots and facula plages on the Sun during the 30-day series did not allow us to study the effect of such magnetic structures on the large-scale flows. In order to test such an influence, we selected a complementary series (12-18 April 2016) with a very large sunspot (NOAA 12529, Fig. 6). This isolated sunspot crossed the central meridian at latitude of $10^{\circ}$ on 13 April 2016 and had the projection-corrected area of

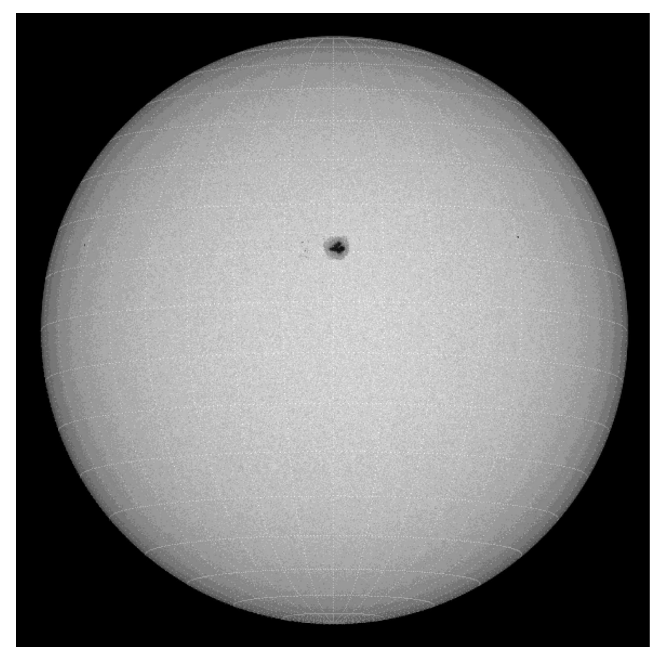

Fig. 6. Context image of the sunspot at the central meridian on 13 April 2016 at 23:00 UT.

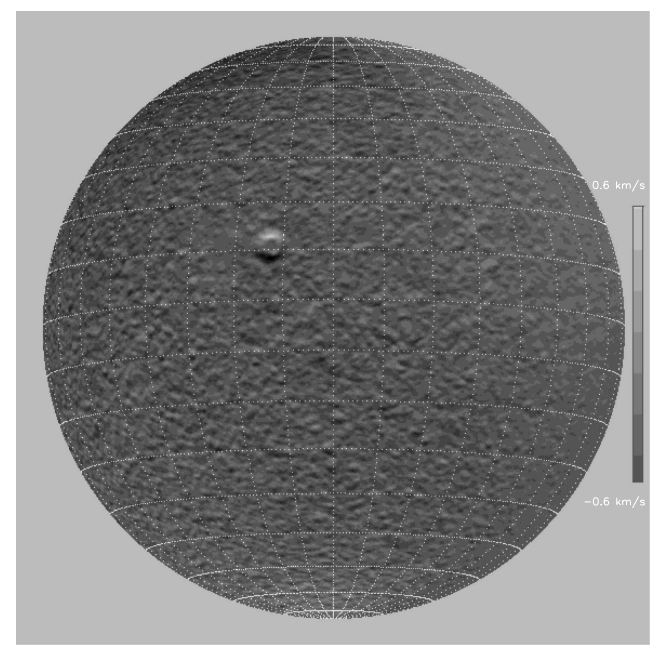

Fig. 7. Image of $v_{\theta}$ on 12 April 2016. Only quiet-Sun regions occupy the central meridian.

960 millionths of solar hemisphere according to the archive of Heliophysical Observatory, Debrecen.

We attempted to study the effects of the presence of magnetised plasma and thus we studied separately three different situations: a quiet-Sun region, the flow field around a sunspot, and a flow field around the plage region following the spot. In this complementary study, we focussed on the velocities determined by the combination of horizontal CST and Doppler velocities only. To capture the characteristic behaviour and avoid possible projection effects, we always studied only a patch around the central meridian and used the solar rotation that carried the regions of interest into this particular patch during the entire sequence.

In this way, all the regions, including quiet, plage, and sunspot regions, were derotated to the central meridian. Then, we applied an averaging in longitude between $-2^{\circ}$ and $12^{\circ}$ for the quiet Sun (Fig. 7) and plage (Fig. 8), and between $-7^{\circ}$ and $7^{\circ}$ for the sunspot (Fig. 9), to have the averaged patch well centred on the specified structure.

Figure 10 shows the daily averages of the zonal velocity for a sequence of 5 days. The dashed line represents the reference of the quiet-Sun rotation obtained for 12 April 2016, for which we 


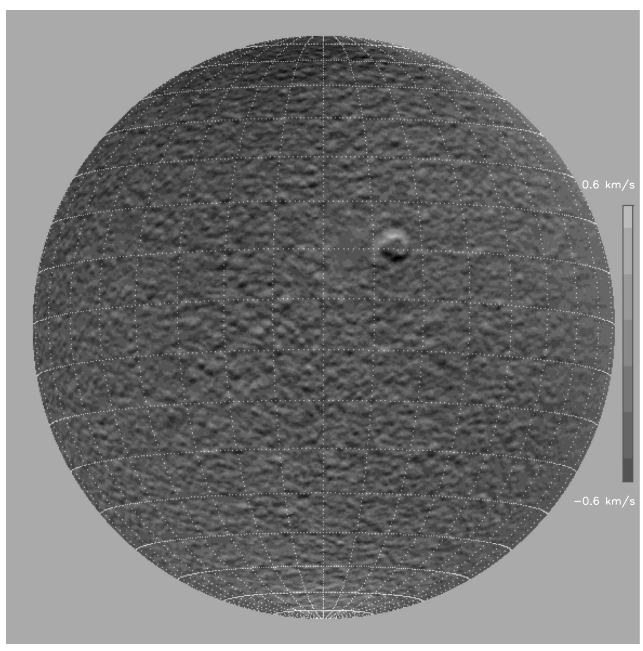

Fig. 8. Image of $v_{\theta}$ on 14 April 2016. The plage region is located at the central meridian.

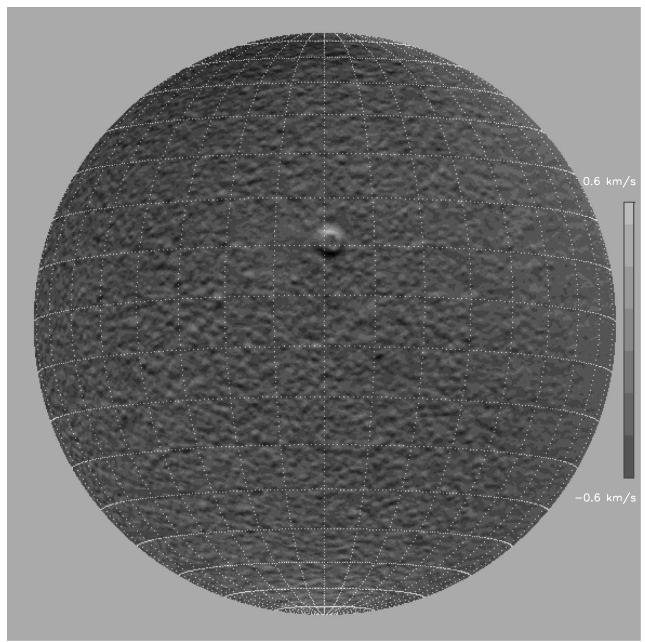

Fig. 9. Image of $v_{\theta}$ on 13 April 2016. The sunspot is positioned at the central meridian.

fit the fourth degree polynomial in $\sin \theta$ as usual. This fit is given in $\mu \mathrm{rad} \mathrm{s}^{-1}$ by the formula

$$
\begin{aligned}
\Omega(\theta)= & 2.75+0.0173 \sin \theta-0.431 \sin ^{2} \theta \\
& +0.280 \sin ^{3} \theta-0.640 \sin ^{4} \theta,
\end{aligned}
$$

giving an equatorial velocity of $1.91 \pm 0.005 \mathrm{~km} \mathrm{~s}^{-1}$. In Fig. 10 the amplitude is normalised to the direct Doppler solar rotation determination of the same day, following the procedure introduced in the previous sections. To avoid overlapping of the curves in Fig. 10, we introduced an artificial shift in amplitude date to date.

At the sunspot latitude $\left(10^{\circ}\right)$ we observe a depression on 14 April, which is related to the presence plage and not to the sunspot, which was at the central meridian a day earlier. That depression is related to a lowered solar rotation which in fact is due to the proper motion of the following faculae plage moving eastward with a mean velocity of $80 \mathrm{~m} \mathrm{~s}^{-1}$. This separation velocity amplitude is compatible with the length of the evolutionary phases (larger than 5 days; see Verma et al. 2016). The solar differential rotation does not appear to be sensitive to largescale sunspot flows because they are largely radially symmetric,

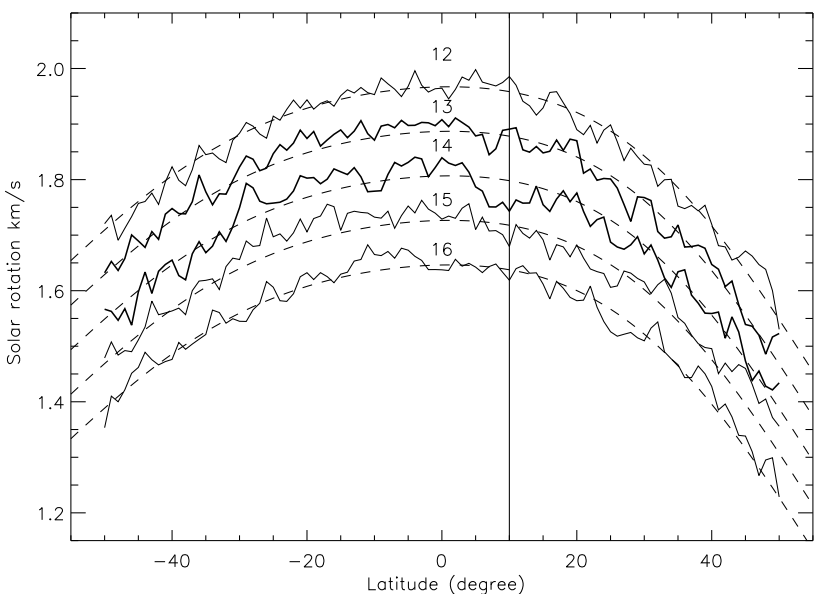

Fig. 10. Solar zonal rotation $\Omega(\theta)$ expressed in $\mathrm{km} \mathrm{s}^{-1}$. For clarity the plots are shifted down in amplitude from date to date.

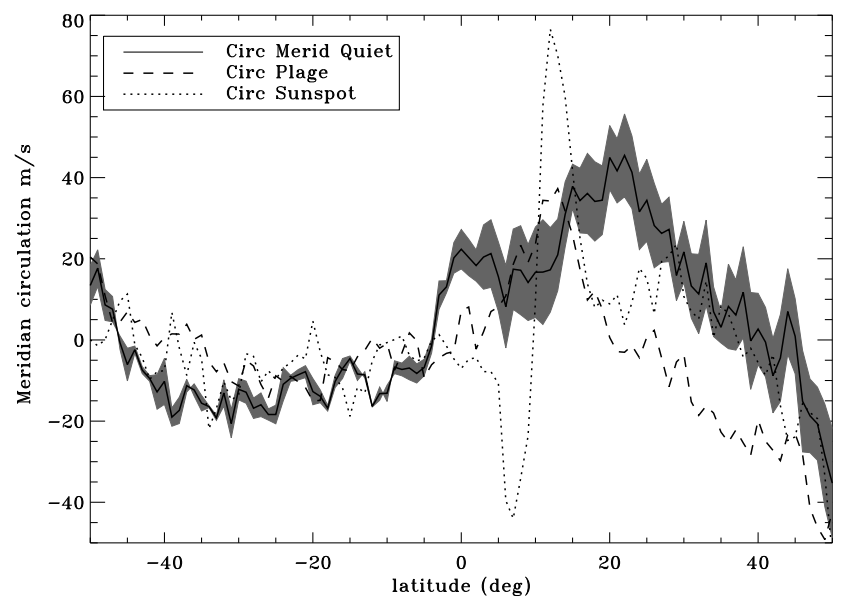

Fig. 11. Meridian circulation expressed in $\mathrm{m} \mathrm{s}^{-1}$ in a quiet Sun and its extrema (grey shaded), sunspot, and plage regions.

thus having opposite directions eastward and westward from the spot and thus practically cancelling each other out.

Figure 11 shows the mean meridional circulation for the quiet-Sun, sunspot, and plage regions which are centred onto the central meridian similar to the differential rotation describe above. In order to get the dispersion of the velocities in the quiet Sun, which is our reference region, the temporal window was fixed to 3 days (like in Fig. 5) and 5 days for plage and sunspot region. The quiet Sun exhibits the classic decreasing behaviour between the northern and southern regions and amplitude in the quiet Sun lies between -25 and $30 \mathrm{~m} \mathrm{~s}^{-1}$. The grey shaded area in Fig. 11 represents the extrema of the meridional circulation for the quiet Sun. The larger dispersion of the meridional circulation in the northern region shows the sensitivity of that signal to the projection effects $\left(B_{0}=-5.72^{\circ}\right)$. The amplitude is larger than determined for the 30-day series (Fig. 5) probably due to the different cycle of activity and projection effects.

The meridional circulation appears to be affected by the sunspot region in which the moat flow modifies greatly the largescale flow with amplitude up to $100 \mathrm{~m} \mathrm{~s}^{-1}$. So during the sunspot life (few days or more) the diffusion of the magnetic elements in the meridional direction are probably affected and must be taken into account in the diffusion model of magnetic field on the Sun surface (Cossette et al. 2017). 


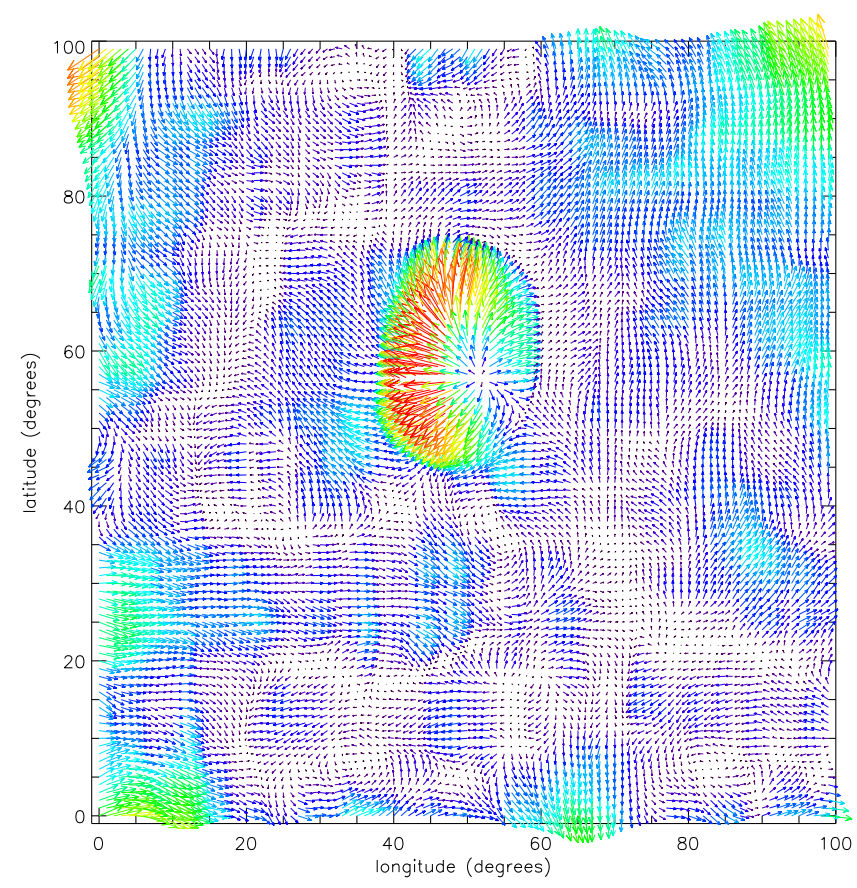

Fig. 12. Flow fields around the sunspot averaged over 5 days and 7 degrees of spatial window.

In case of the plage region we note a similar behaviour to the quiet region in the southern region, but a smaller amplitude in the northern region. We do not know the origin of that amplitude reduction. We question if it is due to the lower amplitude in the plage region, which does not allow the transfer of the regular flow to higher latitudes or to a pure observational coincidence. New observations are required to conclude that point. With only a few day average it is not possible to reliably detect the torsional oscillations in the residual flow as the amplitude of the variations of such residual flow is about $10 \mathrm{~m} \mathrm{~s}^{-1}$, which is larger that the expected magnitude of torsional oscillation of $4 \mathrm{~m} \mathrm{~s}^{-1}$.

Following Löptien et al. (2017) we measured the large-scale flow around the large sunspot. We used the horizontal flows measured in our 5-day sequence, tracked the sunspot location over the whole series, and averaged all flow maps such that they are co-spatial in the location of the spot. Figure 12 shows the flows in which the sunspot moat diverging motions are clearly visible. With that temporal ( 5 day) and spatial $6^{\circ}$ LCT window, we did not observe the large-scale converging flow to the sunspot observed by (Löptien et al. 2017). The amplitude of the measured flows are around $30 \mathrm{~m} \mathrm{~s}^{-1}$ except in the moat where the amplitude goes up to $149 \mathrm{~m} \mathrm{~s}^{-1}$. On that particular sunspot (Löptien et al. 2017) did not observe converging flows towards the sunspot (Löptien, priv. comm.).

\section{Discussion and conclusions}

We carried out CST and helioseismology investigation of solar differential rotation and meridian circulation at the Sun surface on a 30-day HMI/SDO sequence. We completed this study by applying the LCT and the FLCT onto the divergence field obtained from the CST. The usage of various methods allowed us to compare the results. As a complementary study, we also examined the influence of a large sunspot on these large-scale flows with a specific 7-day HMI/SDO sequence.

We find that the large-scale flows measured by the CST on the solar surface and the same flow determined with the same data with the helioseismology in the first $1 \mathrm{Mm}$ below the surface, are in good agreement in amplitude and direction. The torsional waves are also located at the same latitudes with amplitude of the same order. Using the CST method we are able to measure the meridional circulation correctly with only 3 days of data after averaging between $\pm 15^{\circ}$ in longitude. This indicates to us the minimal number of days necessary to follow correctly the evolution of the large-scale solar flows.

The application of the LCT on the divergence maps does not show the same differential rotation as the Doppler measurement. The FLCT applied also to the same data gives a result closer to the Doppler measurement but still shows lower amplitude for the high latitudes. Doppler is a direct determination of the velocities on the Sun, while LCT, FLCT, and CST are indirect velocity determination. In addition, LCT and FLCT do not measure the same flows as those determined by the Doppler. The LCT and FLCT are sensitive to the granules and intergranules horizontal displacements but Doppler is affected by its distance to the disc centre (vertical motion at the disc centre and horizontal motion elsewhere). Thus a detailed comparison of the large-scale flows obtained by the various methods (LCT, FLCT, CST, and Doppler) is still delicate and can reflect different properties of the turbulent flows of the photosphere.

The differential rotation and meridional circulation was determined by examining large flows in different activity regions: quiet Sun, sunspot, and plage. The differential rotation is sensitive to the relative separation motion between the leading sunspot and following plage. The meridional circulation is sensitive to the moat flow, which clearly modifies its amplitude locally and probably to surrounding latitudes.

Acknowledgements. The SDO data are courtesy of NASA and the SDO/HMI science team. This work was granted access to the HPC resources of CALMIP under the allocation 2011-[P1115]. This work was supported by the CNRS Programme National Soleil Terre. M. Š. is supported by the project RVO:67985815. The authors wish to thank the anonymous referee for very helpful comments and suggestions that improved the quality of the manuscript.

\section{References}

Basu, S., \& Antia, H. M. 2010, ApJ, 717, 488

Cossette, J.-F., Charbonneau, P., Smolarkiewicz, P. K., \& Rast, M. P. 2017, ApJ, 841,65

Duvall, Jr., T. L. 1979, Sol. Phys., 63, 3

Duvall, Jr., T. L., Jefferies, S. M., Harvey, J. W., \& Pomerantz, M. A. 1993, Nature, 362, 430

Fisher, G. H., \& Welsch, B. T. 2008, in Subsurface and Atmospheric Influences on Solar Activity, eds. R. Howe, R. W. Komm, K. S. Balasubramaniam, \& G. J. D. Petrie, ASP Conf. Ser., 383, 373

Fleck, B., Couvidat, S., \& Straus, T. 2011, Sol. Phys., 271, 27

Gizon, L., \& Rempel, M. 2008, Sol. Phys., 251, 241

Hathaway, D. H. 1987, Sol. Phys., 108, 1

Hathaway, D. H. 1996, ApJ, 460, 1027

Hathaway, D. H. 2012, ApJ, 760, 84

Hathaway, D. H., \& Upton, L. 2014, J. Geophys. Res. (Space Phys.), 119, 3316

Howard, R., \& Harvey, J. 1970, Sol. Phys., 12, 23

Howard, R., \& Labonte, B. J. 1980, ApJ, 239, L33

Howe, R. 2009, Liv. Rev. Sol. Phys., 6, 1

Howe, R., Christensen-Dalsgaard, J., Hill, F., et al. 2005, ApJ, 634, 1405

Howe, R., Komm, R., Hill, F., et al. 2006, Sol. Phys., 235, 1

Jackiewicz, J., Gizon, L., Birch, A. C., \& Duvall, Jr., T. L. 2007, ApJ, 671, 1051

Javaraiah, J. 2013, Sol. Phys., 287, 197

Komm, R., Howe, R., González Hernández, I., \& Hill, F. 2014, Sol. Phys., 289, 3435

Kosovichev, A. G., \& Zhao, J. 2016, in Lect. Notes Phys., eds. J.-P. Rozelot, \& C. Neiner (Berlin: Springer Verlag), 914, 25

Li, K. J., Shi, X. J., Xie, J. L., et al. 2013, MNRAS, 433, 521

Liang, Z.-C., Birch, A. C., Duvall, Jr., T. L., Gizon, L., \& Schou, J. 2017, A\&A, 601, A46 
Th. Roudier et al.: Large-scale photospheric motions determined from granule tracking from SDO/HMI data

Löptien, B., Birch, A. C., Duvall, T. L., Gizon, L., \& Schou, J. 2016, A\&A, 590, A130

Löptien, B., Birch, A. C., Duvall, Jr., T. L., et al. 2017, A\&A, 606, A28

November, L. 1986, J. Appl. Opt., 25, 392

Paternò, L. 2010, Ap\&SS, 328, 269

Rieutord, M., Roudier, T., Roques, S., \& Ducottet, C. 2007, A\&A, 471, 687

Rincon, F., Roudier, T., Schekochihin, A. A., \& Rieutord, M. 2017, A\&A, 599, A69

Roudier, T., Rieutord, M., Malherbe, J. M., et al. 2012, A\&A, 540, A88

Roudier, T., Rieutord, M., Prat, V., et al. 2013, A\&A, 552, A113

Scherrer, P. H., Schou, J., Bush, R. I., et al. 2012, Sol. Phys., 275, 207

Schou, J., Scherrer, P. H., Bush, R. I., et al. 2012, Sol. Phys., 275, 229

Sudar, D., Brajša, R., Skokić, I., Poljančić Beljan, I., \& Wöhl, H. 2017, Sol. Phys. 292, 86

Ulrich, R. K. 2010, ApJ, 725, 658
Švanda, M., Klvaňa, M., \& Sobotka, M. 2006, A\&A, 458, 301

Švanda, M., Klvaňa, M., Sobotka, M., \& Bumba, V. 2008, A\&A, 477, 285

Švanda, M., Gizon, L., Hanasoge, S. M., \& Ustyugov, S. D. 2011, A\&A, 530, A148

Švanda, M., Roudier, T., Rieutord, M., Burston, R., \& Gizon, L. 2013, ApJ, 771, 32

Verma, M., Steffen, M., \& Denker, C. 2013, A\&A, 555, A136

Verma, M., Denker, C., Balthasar, H., et al. 2016, A\&A, 596, A3

Welsch, B. T., Fisher, G. H., Abbett, W. P., \& Regnier, S. 2004, ApJ, 610, 1148

Zhao, J. 2016, Adv. Space Res., 58, 1457

Zhao, J., \& Kosovichev, A. G. 2004, ApJ, 603, 776

Zhao, J., Bogart, R. S., Kosovichev, A. G., Duvall, Jr., T. L., \& Hartlep, T. 2013 ApJ, 774, L29

Zhao, J., Kosovichev, A. G., \& Bogart, R. S. 2014, ApJ, 789, L7 